\title{
A novel protein encoded by a circular RNA circPPP1R12A promotes tumor pathogenesis and metastasis of colon cancer via Hippo-YAP signaling
}

Xiao Zheng ${ }^{1,2,3}$, Lujun Chen ${ }^{1,2,3}$, You Zhou ${ }^{1,2,3}$, Qi Wang ${ }^{1,2,3}$, Zhuojun Zheng ${ }^{2,4}$, Bin Xu ${ }^{1,2,3}$, Chen Wu ${ }^{2,3,5}$, Qi Zhou ${ }^{5}$, Wenwei $\mathrm{Hu}^{1,2,5}$, Changping $\mathrm{Wu}^{1,2,5}$ and Jingting Jiang ${ }^{1,2,3^{*}}$

\begin{abstract}
Background: It has been well established that circular RNAs (circRNAs) play an important regulatory role during tumor progression. Recent studies have indicated that even though circRNAs generally regulate gene expression through miRNA sponges, they may encode small peptides in tumor pathogenesis. However, it remains largely unexplored whether circRNAs are involved in the tumorigenesis of colon cancer (CC).
\end{abstract}

Methods: The expression profiles of circRNAs in CC tissues were assessed by circRNA microarray. Quantitative realtime $P C R$, RNase $R$ digestion assay and tissue microarray were used to confirm the existence and expression pattern of circPPP1R12A. The subcellular distribution of circPPP1R12A was analyzed by nuclear mass separation assay and fluorescence in situ hybridization (FISH). SDS-PAGE and LC/MS were employed to evaluate the protein-coding ability of circPPP1R12A. CC cells were stably transfected with lentivirus approach, and cell proliferation, migration and invasion, as well as tumorigenesis and metastasis in nude mice were assessed to clarify the functional roles of circPPP1R12A and its encoded protein circPPP1R12A-73aa. RNA-sequencing and Western blotting analysis were furthered employed to identify the critical signaling pathway regulated by circPPP1R12A-73aa.

Results: We firstly screened the expression profiles of human circRNAs in CC tissues and found that the expression of hsa_circ_0000423 (termed as circPPP1R12A) was significantly increased in CC tissues. We also found that circPPP1R12A was mostly localized in the cytoplasm of CC cells. Kaplan-Meier analysis showed that patients with higher levels of circPPP1R12A had a significantly shorter overall survival. By gain- and loss-of-function approaches, the results suggested that cirCPPP1R12A played a critical role in proliferation, migration and invasion of CC cells. Furthermore, we showed that circPPP1R12A carried an open reading frame (ORF), which encoded a functional protein (termed as circPPP1R12A-73aa). Next, we found that PPP1R12A-C, not circPPP1R12A, promoted the proliferation, migration and invasion abilities of CC in vitro and in vivo. Finally, we identified that circPPP1R12A-73aa promoted the growth and metastasis of CC via activating Hippo-YAP signaling pathway. In addition, the YAP specific inhibitor Peptide 17 dramatically alleviated the promotive effect of circPPP1R12A-73aa on CC cells.

(Continued on next page)

\footnotetext{
*Correspondence: jiangjingting@suda.edu.cn

'Department of Tumor Biological Treatment, the Third Affiliated Hospital of

Soochow University, Changzhou 213003, People's Republic of China

${ }^{2}$ Jiangsu Engineering Research Center for Tumor Immunotherapy,

Changzhou 213003, People's Republic of China

Full list of author information is available at the end of the article
}

(c) The Author(s). 2019 Open Access This article is distributed under the terms of the Creative Commons Attribution 4.0 International License (http://creativecommons.org/licenses/by/4.0/), which permits unrestricted use, distribution, and reproduction in any medium, provided you give appropriate credit to the original author(s) and the source, provide a link to the Creative Commons license, and indicate if changes were made. The Creative Commons Public Domain Dedication waiver (http://creativecommons.org/publicdomain/zero/1.0/) applies to the data made available in this article, unless otherwise stated. 
(Continued from previous page)

Conclusions: In the present study, we illustrated the coding-potential of circRNA circPPP1R12A in the progression of CC. Moreover, we identified that circPPP1R12A-73aa promoted the tumor pathogenesis and metastasis of CC via activating Hippo-YAP signaling pathway. Our findings might provide valuable insights into the development of novel potential therapeutic targets for CC.

Keywords: Colon cancer, Circular RNA (circRNA), Protein coding, Proliferation, Metastasis

\section{Introduction}

Colon cancer (CC) is a common type of malignant tumor worldwide, which is the third frequently detected cancer and the fourth leading cause of cancer-related death [1]. Although the therapeutic approaches for CC have been greatly improved, more than half of CC patients ultimately die. Therefore, it is urgently necessary to explore other unknown mechanisms underlying the progression of CC [2-4].

With the rapid development of next-generation sequencing technologies, more and more previously unknown transcripts have been identified. Non-coding RNAs (ncRNAs), as most of above-mentioned transcripts, can not be translated into proteins due to the lack of obvious open reading frames (ORFs) in organisms [5-8]. Circular RNAs (circRNAs), a class of circularly configured RNA molecules consisting of an upstream splice acceptor and a downstream splice donor [9], have been recently reported to be widely spread in eukaryotes. There are three types of circRNAs according to their origins, including exonic, intronic and intergenic regions. The exonic circRNAs (originated from exonic sequences) typically reside in the cytoplasm, while intronic circRNAs (intron-retaining circRNAs) mostly remain in nuclei [10]. The exonic circRNAs, the end-products of splicing, are the most studied circRNAs [11]. Due to their abundance and stability, it is well established that certain circRNAs can widely regulate biological activities by functioning as ceRNA or regulators of RNA binding protein in modulating protein-gene transcription [12-15]. Recently, several circRNAs have been found to encode proteins [16-18]. However, it remains unclear whether there are protein-coding circRNAs involved in CC tumorigenesis, and their functional products are still elusive.

In the present study, we identified an up-regulated circRNA hsa_circ_0000423 (termed as circPPP1R12A) in CC tissues. Silencing of circPPP1R12A by siRNAs suppressed the proliferation ability of $\mathrm{CC}$ cells, while the migration and invasion abilities of $\mathrm{CC}$ cells remained unaffected. Moreover, a 73-amino acid (aa) protein encoded by circPPP1R12A was identified by overlapping genetic codes and proteomics. The in vitro and in vivo data showed that when the protein-coding ability of circPPP1R12A was abolished by mutating the start codon, its promotive effect on cell proliferation was reversed.
Moreover, we identified that circPPP1R12A-73aa promoted the growth and metastasis of $\mathrm{CC}$ via activating Hippo-YAP signaling pathway. Collectively, a protein encoded by circRNA circPPP1R12A contributed to the rapid proliferation of CC cells.

\section{Materials and methods}

\section{Patients and tissue samples}

A total of 20 paired human CC and adjacent non-tumor tissues were collected between 2014 and 2017 at the Third Affiliated Hospital of Soochow University. This study was approved by the Ethical Committee of the Third Affiliated Hospital of Soochow University, and the written informed consent was provided by each participant prior to surgery.

\section{Expression profile analysis of circRNAs}

The circRNA microarray (Arraystar Human circRNAs chip, ArrayStar) consisting of more than 5000 probes specific to splicing sites of human circRNAs was used. After hybridization, 20 pairs of CC samples (tumor tissues and paired adjacent non-tumor tissues) were examined using the circRNA micro-array provided by Kangcheng Bio-Tech Inc.

\section{Cell lines and cell culture}

Human CC cell lines HT-29, HCT-116, SW480, SW620, LoVo, SW48, DLD-1, Caco2 and HCT-15) and normal human colon mucosal epithelial cell line NCM460 were obtained from Chinese Academy of Sciences, Shanghai Institutes for Biological Sciences. All these cell lines were maintained in DMEM supplemented with $10 \%$ FBS under standard culture conditions $\left(5 \% \mathrm{CO}_{2}, 37^{\circ} \mathrm{C}\right)$.

RNA extraction, gDNA extraction, and quantitative realtime PCR analysis

RNA extraction and quantitative real-time PCR were performed as previously described [19]. Genomic DNA (gDNA) was extracted from tissues using PureLink Genomic DNA Mini Kit according to the manufacturer's instructions (Thermo Fisher Scientific, K182001). The real-time PCR was conducted using the ABI Vii7 system (Applied Biosystems, USA), and SYBR Green was used as a DNA-specific fluorescent dye. Human GAPDH was selected as a housekeeping gene. Primers were 
synthesized as follows, the human circPPP1R12A forward primer: 5'-ACAGCAGCAGGCTAGAAAAG-3', and reverse primer: 5'-TGTCCTAAGCAGGAAAAAC A-3'; GAPDH forward: 5'-TGACTTCAACAGCGACA CCCA-3', and GAPDH reverse: 5'-CACCCTGTTGC TGTAGCCAAA-3'. Relative gene expression was calculated by the comparative $C T$ method $(\triangle \Delta C T)$, where the fold enrichment was determined as: $2^{-[\Delta \mathrm{CT}}$ (sample) $-\Delta \mathrm{CT}$ (calibrator)]

\section{RNA in situ hybridization (ISH) and fluorescence in situ hybridization (FISH)}

ISH and FISH were carried out to detect the circPPP1R12A expression in colon tissue microarray (Shanghai Outdo Biotech, Shanghai, China) using digoxigenin- or FITC-labeled probe as previously reported [20]. After stained with an anti-digoxin or anti-FITC $\mathrm{mAb}$ (Roche Applied Science), the sections were incubated in the presence or absence of NBT/BCIP in the dark, mounted and examined under microscope.

\section{Plasmids, siRNAs and cell transfection}

Human circPPP1R12A over-expressing vector (Lv-circPPP1R12A) and the control plasmid (pLVX-IRES-GFP) were purchased from Genelily Biotechnology Company (Shanghai, China). The expression of circPPP1R12A was transiently silenced by small interference RNAs (siRNAs) specific to human circPPP1R12A, which were generated by GenePharma (GenePharma Corporation, Shanghai, China). The experiment was divided into several groups as follows: si-NC (transfected with siRNA NC vector) group, si-circPPP1R12A (transfected with circPPP1R12A siRNA) group, OE-NC (transfected with over-expression NC vector) group and OE-circPPP1R12A (transfected with circPPP1R12A over-expression vector) group. The above-mentioned vectors were transfected by Lipofectamine $^{\circ} 3000$ transfection reagent as previously reported. After transfection for $48 \mathrm{~h}$, the transfection efficiency of cells in each group was assessed by real-time PCR analysis.

\section{Cell proliferation, colony formation and cell cycle assays}

Cell growth was monitored by CCK8 assay as previously reported [21]. Colony formation assays were performed to indicate the cloning capability of CC cells. For cell cycle assay, subG1, S and G2 peaks were detected from the propidium iodide-stained CC cells by flow cytometry (BD FACS Canto II, BD Bioscience, NJ, USA) and analyzed using the Modfit software.

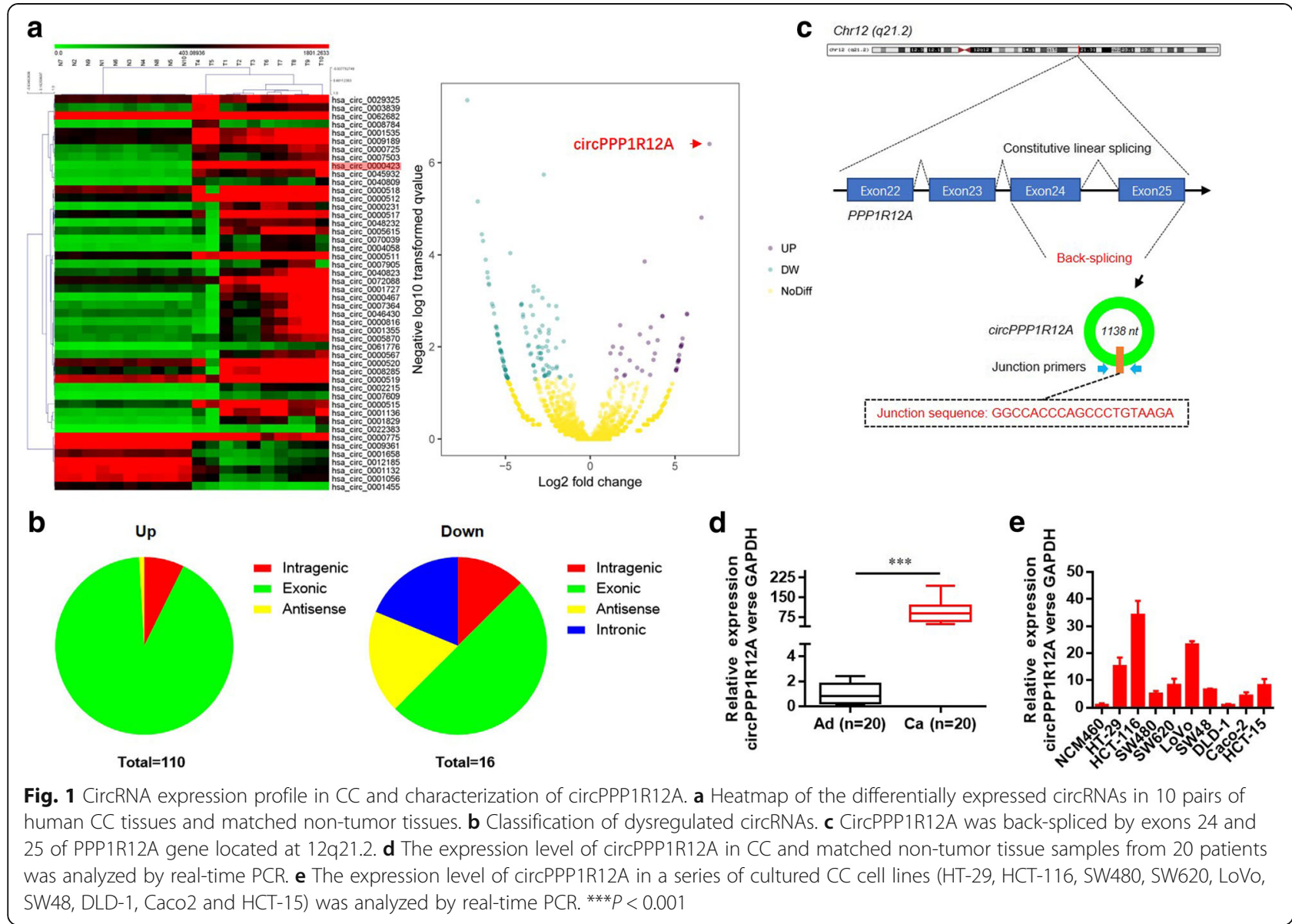




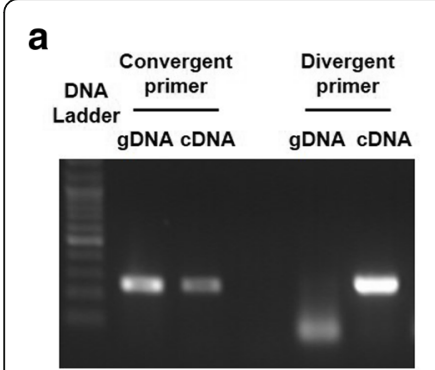

b

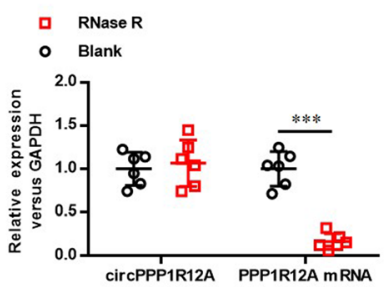

e
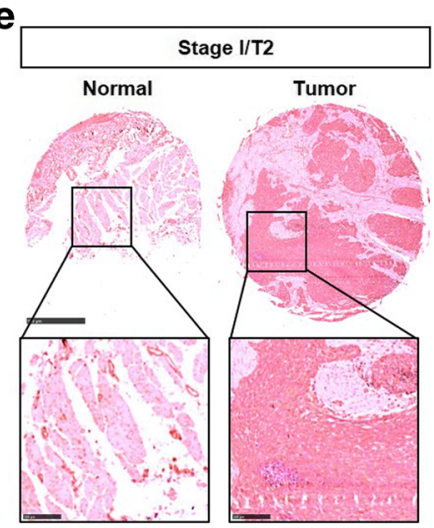

C

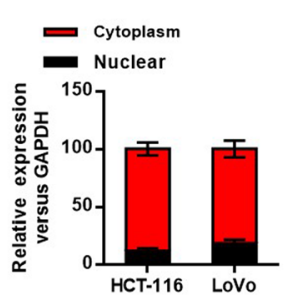

d

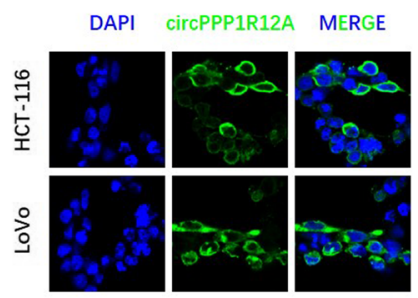

Fig. 2 Characterization the existence and subcellular distribution of circPPP1R12A in CC cells and tissues. a The divergent primers detected circPPP1R12A in CDNA but not in gDNA. b Real-time PCR analysis of circPPP1R12A and linear PPP1R12A mRNA after treatment with RNase R in HCT-116 cells showed that circPPP1R12A was resistant to RNase R treatment. The sub-cellular distribution of circPPP1R12A was mostly present in the cytoplasm by the nuclear mass separation assay (c) and FISH (d). e The level of circPPP1R12A was analyzed by in situ hybridization on CC tissue microarray, showing that circPPP1R12A was up-regulated in CC tissues compared with normal tissues, and such up-regulation was positively correlated with larger tumors and a higher TNM stage. f Kaplan-Meier analysis of the correlation between circPPP1R12A expression and overall survival showed that patients with higher levels of circPPP1R12A had a significantly shorter overall survival. ${ }^{* *} P<0.001$

\section{Migration and invasion assay}

The cell migration ability was assessed using wound-healing assay as previously reported. The invasion assay was analyzed by transwell chamber with $8-\mu$ m pores (BD Falcon, Franklin Lakes, NJ, USA).

\section{Western blotting analysis}

Western blotting analysis was performed as previously described [19] with antibodies against flag and GAPDH (Abcam, Cambridge, MA, USA). GAPDH was used as an endogenous control to normalize the protein loading.

\section{Analysis of peptide patterns by LC-MS/MS}

The protein identification by LC-MS/MS was performed according to previously reported method [22]. Briefly, equal amounts of proteins $(50 \mu \mathrm{g})$ were subjected to $12 \%$ SDS-PAGE. The protein bands near $10 \mathrm{kDa}$ were excised according to size and chopped into $1 \mathrm{~mm}^{3}$ pieces. Finally, peptide mixtures were extracted from the gels and dried prior to LC-MS/MS analysis.

\section{Statistical analysis}

Statistical analyses were completed using the SPSS version 16.0 (SPSS Inc., Chicago, IL, USA). Survival analysis
Table 1 The detailed clinical parameters of the 100 patients

\begin{tabular}{lc}
\hline Clinical Parameters & Cases \\
\hline Gender & 76 \\
Male & 24 \\
Female & \\
Age (years) & 65 \\
$<55$ & 35 \\
$\geq 55$ & \\
Tumor size (cm) & 46 \\
$\leq 5$ & 54 \\
$>5$ & \\
Pathological stage & 42 \\
I + II & 58 \\
III & \\
Pathological type & 24 \\
Massive & 76 \\
Nodular & \\
T stage & 27 \\
I+ II & 73 \\
III + IV & \\
\hline
\end{tabular}


Table 2 Univariate and multivariate survival analysis of CC patients from SEER database

\begin{tabular}{|c|c|c|c|c|}
\hline \multirow[t]{2}{*}{ Variable } & \multicolumn{2}{|l|}{ Univariate analysis } & \multicolumn{2}{|l|}{ Multivariate analysis } \\
\hline & $\mathrm{HR}(95 \% \mathrm{Cl})$ & $P$ & HR $(95 \% \mathrm{Cl})$ & $P$ \\
\hline Sex (Male vs. Female) & $0.935(0.543-1.611)$ & 0.809 & & \\
\hline Age (> 55 vs. $16-55$ years) & $1.620(0.584-4.495)$ & 0.354 & & \\
\hline Tumor size (cm) (> 5 vs. $0-5)$ & $1.007(0.562-1.802)$ & 0.982 & & \\
\hline Pathological stage (III vs. I + II) & $2.815(1.520-5.215)$ & 0.001 & $2.865(1.521-5.394)$ & 0.001 \\
\hline Pathological type (Massive vs. Nodular) & $1.565(0.907-2.701)$ & 0.108 & & \\
\hline circPPP1R12A ISH Score (3-4 vs. 0-2) & $2.788(1.484-5.237)$ & 0.001 & $2.927(1.574-5.443)$ & 0.001 \\
\hline
\end{tabular}

Abbreviation: $I S H$, In Situ Hybridization

was carried out using the Kaplan-Meier method, and the log-rank test was used to compare the survival curves. Other data were assessed with Student's two-tailed t-test by GraphPad Prism 5.0 software package (GraphPad Software, Inc., San Diego, USA). A $P$ value $<0.05$ was considered as statistically significant.

\section{Results}

\section{Expression profiles and screening of circRNAs in CC} tissues and cells

Firstly, circRNA microarray was employed to characterize the expression profiles of circRNAs in paired CC tissues and adjacent non-tumor tissues from 10 patients. A total of 126 circRNAs $(P<0.05$ and fold change $>1.5$ ) were differentially expressed between the
CC tissues and paired adjacent non-tumor tissues. Among the 126 differentially expressed circRNAs, 110 circRNAs were up-regulated, while 16 ones were down-regulated in CC tissues compared with the adjacent non-tumor tissues (Fig. 1 a). Additional file 1: Table S1 lists the detailed information about these dysregulated circRNAs. These circRNAs were mostly located at exonic regions (Fig. $1 \mathrm{~b}$ ). As the most up-regulated circRNA, hsa_circ_0000423 (termed as circPPP1R12A) was back-spliced of exons 24/25 of PPP1R12A gene located at 12 q21.2 (Fig. $1 \mathrm{c}$ ). Next, we re-examined the expression of circPPP1R12A in CC and paired non-tumor tissue samples from 20 patients by quantitative real-time PCR to confirm its elevated expression (Fig. 1 d). We further found that the circPPP1R12A expression was

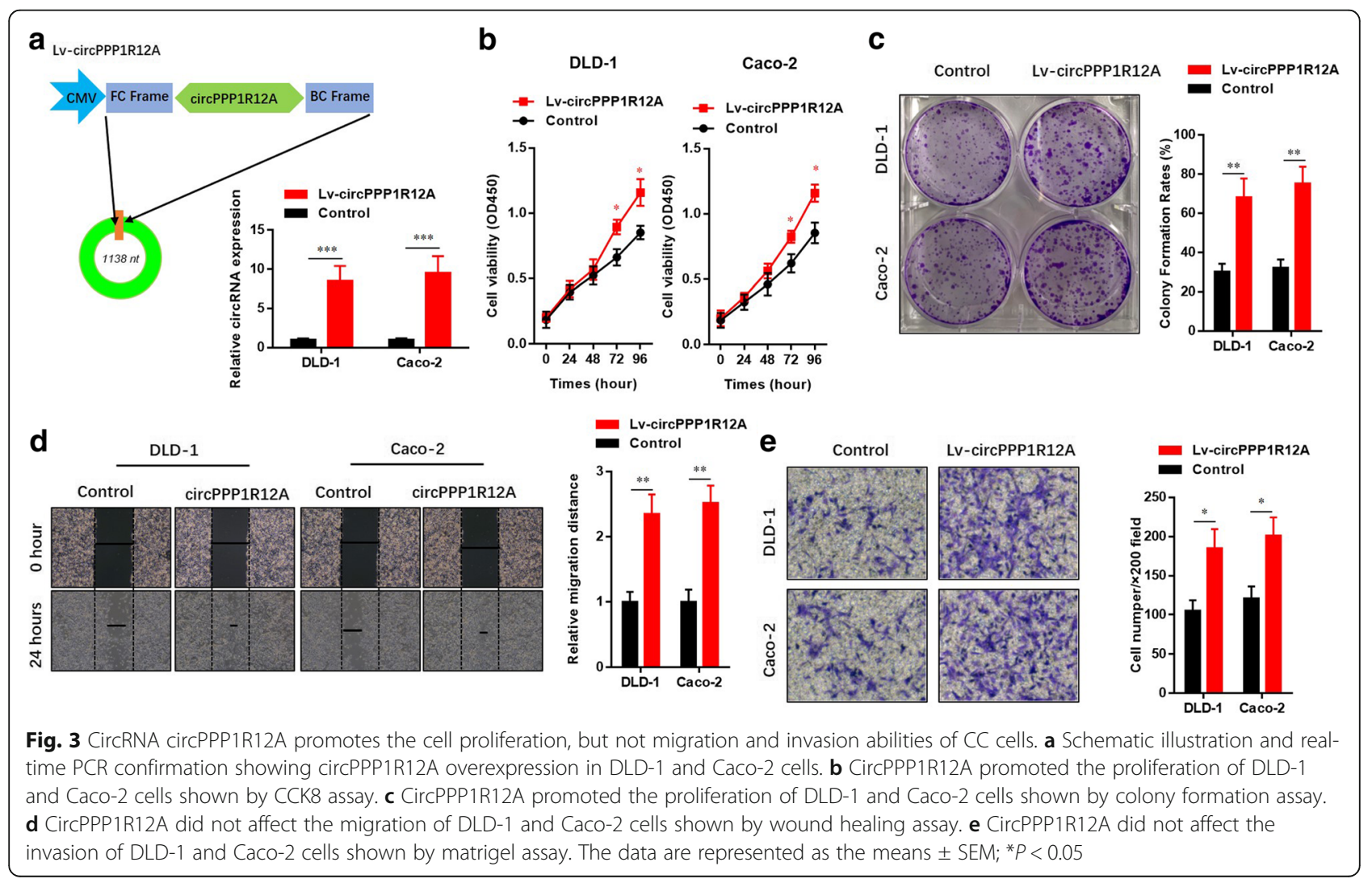




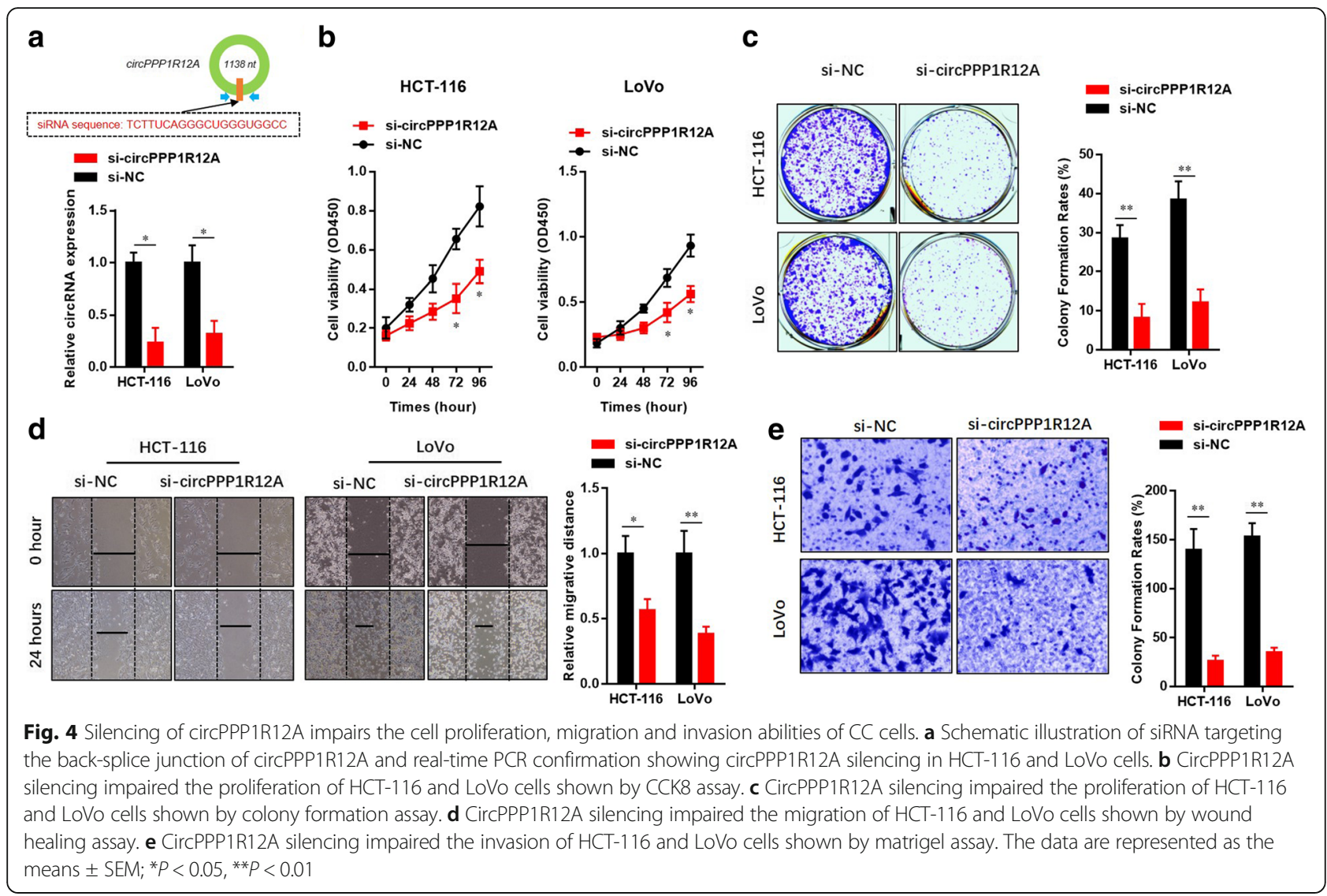

consistently and significantly increased in CC tissues compared with the matched controls, while the expression of PPP1R12A (linear transcript of PPP1R12A gene) was comparable in $\mathrm{CC}$ tissues and matched controls (Additional file 2: Figure S1a). Moreover, the expression of circPPP1R12A was significantly up-regulated in a series of cultured CC cell lines (HT-29, HCT-116, SW480, SW620, LoVo, SW48, DLD-1, Caco2 and HCT-15) compared with a normal human colon mucosal epithelial cell line NCM460 cells. The highest expression of circPPP1R12A was found in HCT-116 cells, followed by LoVo cells (Fig. 1 e). Therefore, our subsequent experiments focused on the role of circPPP1R12A in CC progression.

\section{Characterization of the existence and subcellular distribution of circPPP1R12A in CC cells and tissues}

In the present study, we designed two sets of primers to characterize circPPP1R12A. One pair (divergent primers) was used to amplify the circular transcripts, while the other pair (convergent primers) was used to detect the linear transcripts. The results suggested that the circular form could be amplified using the convergent primers from both cDNA and gDNA, while it was only amplified from cDNA by divergent primers (Fig. 2 a). To further confirm the existence of circPPP1R12A, the RNase R degradation assay was used to evaluate the resistance of circPPP1R12A to RNase R treatment. Figure $2 \mathrm{~b}$ shows that the linear transcripts of PPP1R12A were degraded by RNase R treatment, while such treatment failed to degrade the circular transcripts of circPPP1R12A. Nuclear mass separation assay (Fig. 2 c) and FISH analysis (Fig. 2 d) reveled that over 93\% of circPPP1R12A appeared in the cytoplasm of HCT-116 and LoVo cells. We also detected the expression of circPPP1R12A in CC tissues by ISH using TMA consisting of 100 pairs of $C C$ and adjacent non-tumor tissues (Fig. 2e). Table 1 lists the detailed clinical parameters of these patients. Among the clinicopathological variables, pathological stage and circPPP1R12A ISH score were identified as risk factors for predicting overall survival based on univariate analysis, while multivariate analysis with Cox regression model further confirmed that pathological stage III and circPPP1R12A ISH score 3-4 were the independent poor prognostic factors (Table 2). Kaplan-Meier survival curves showed that patients with higher expression of circPPP1R12A had a shorter overall survival [HR $=1.886$; $95 \%$ confidence interval (CI), 1.129-3.1529; $P=0.0154$; Fig. 2 f].

CircRNA circPPP1R12A promotes the cell proliferation, but not migration and invasion abilities of CC cells As unlimited cell proliferation, migration and invasiveness are hallmarks of malignant tumor, we next explored 


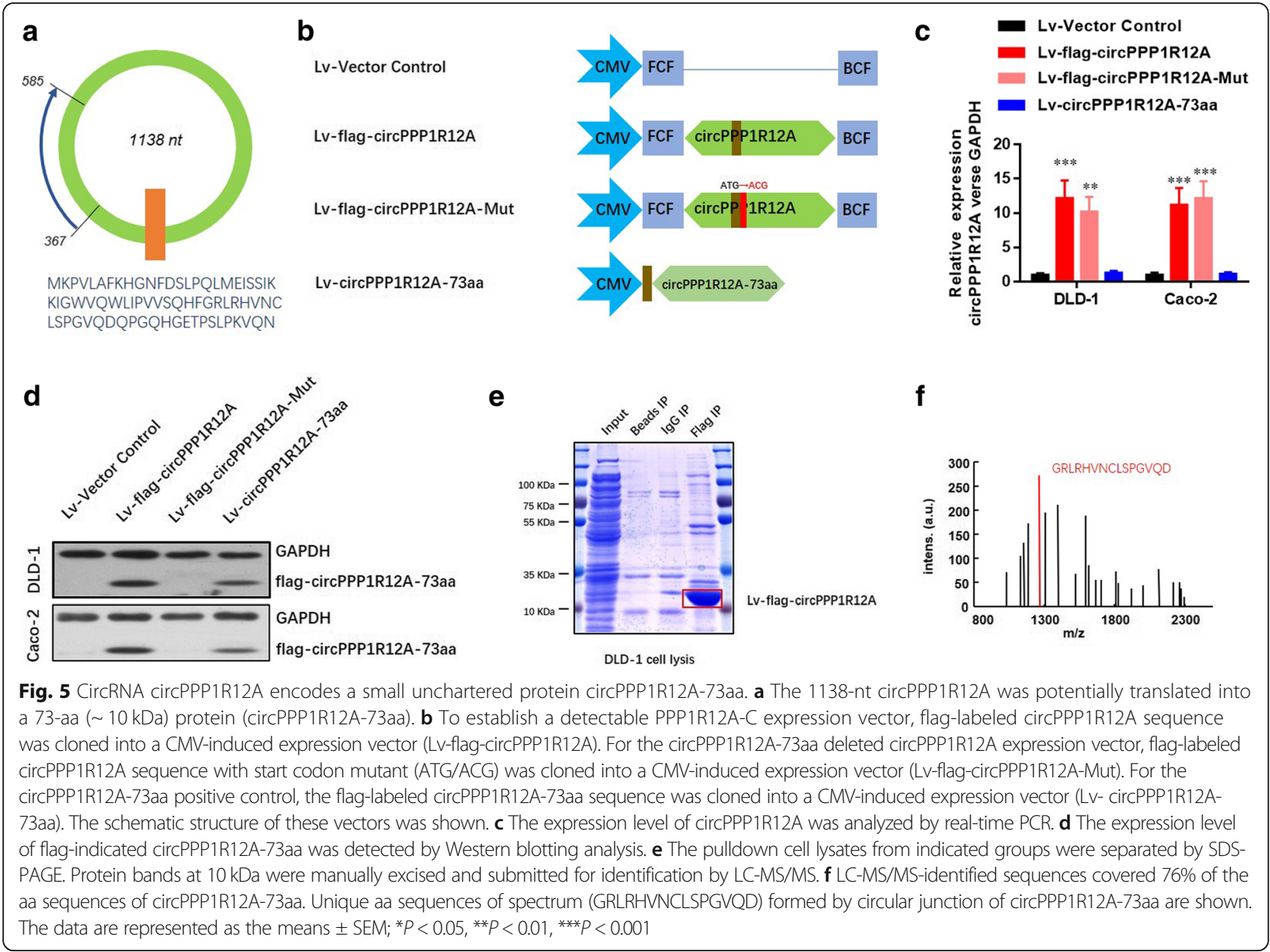

the role of circPPP1R12A in progression of CC by gainand loss-of-function approaches. We overexpressed circPPP1R12A by artificial plasmids in two (DLD-1 and Caco-2) CC cell lines with low circPPP1R12A expression, and the overexpression efficiency was confirmed by real-time PCR (Fig. 3 a). CCK8 assay (Fig. 3 b) and colony formation assay (Fig. $3 \mathrm{c}$ ) indicated that circPPP1R12A overexpression accelerated the cell proliferation of DLD-1 and Caco-2 cells. Subsequently, we explored the effect of circPPP1R12A on motility of CC cells. Wound-healing assay and invasion assay showed that circPPP1R12A overexpression also enhanced the migration (Fig. $3 \mathrm{~d}$ ) and invasion (Fig. 3 e) abilities of DLD-1 and Caco- 2 cells. Moreover, we overexpressed circPPP1R12A by artificial plasmids in two (HCT-116 and LoVo) CC cell lines with high circPPP1R12A expression. Similarly, circPPP1R12A overexpression accelerated the cell proliferation of HCT-116 and LoVo cells, evidenced by CCK8 assay (Additional file 3: Figure S2a) and colony formation assay (Additional file 3: Figure S2b). However, circPPP1R12A overexpression failed to alert the migration (Additional file 3: Figure S2c) and invasion (Additional file 3:
Figure S2d) abilities of DLD and Caco-2 cells in wound-healing assay and invasion assay.

\section{Silencing of circPPP1R12A suppresses the cell} proliferation, migration and invasion abilities of CC cells We next investigated whether silencing of circPPP1R12A affected cell proliferation, migration and invasiveness. The silencing efficiency was confirmed by real-time PCR (Fig. 4 a). As expected, silencing of circPPP1R12A affected the proliferation (Fig. $4 \mathrm{~b}$ ) and colony forming abilities (Fig. $4 \mathrm{c}$ ) of both cell lines. Furthermore, in wound-healing assay and invasion assay, silencing of circPPP1R12A significantly affected the migration (Fig. $4 \mathrm{~d}$ ) and invasion (Fig. 4 e) abilities of HCT-116 and LoVo cells. Collectively, these results suggested that circPPP1R12A played a critical role in proliferation, migration and invasion of CC cells.

\section{CircRNA circPPP1R12A encodes a small uncharacterized protein}

As circRNA circPPP1R12A is originally annotated in circRNA Db database, we found a short 216-nt small ORF 


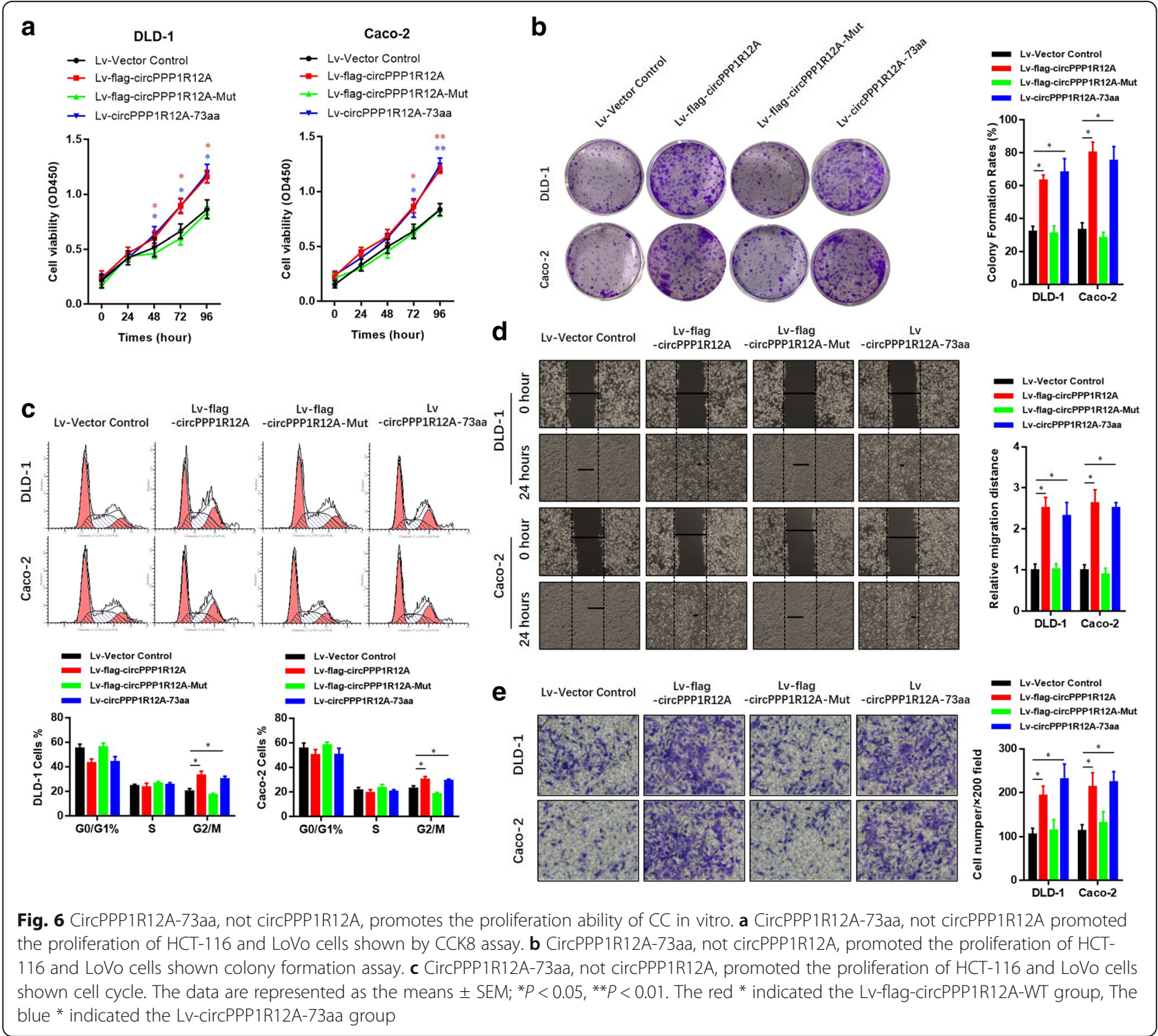

with the potential to encode a conserved 73-aa peptide (Fig. 5 a). Especially, in human circPPP1R12A, the circularization created the tandem start codon 'AUG, which started the translation in combination with overlapping genetic codes. The ORF of circPPP1R12A was supposed to be translated into a putative 73-aa protein (we termed 'circPPP1R12A-73aa'), in which the unique aa sequences were formed (Fig. 5 a). To dissociate the role of circPPP1R12A-73aa from circPPP1R12A, we constructed several flag-labeled vectors for circPPP1R12A (Fig. 5 b). Transfection with the Lv-flag-circPPP1R12A vector and Lv-flag-circPPP1R12A-Mut vector both successfully resulted in the overexpression of cirCPPP1R12A, while transfection with the control vector or Lv-circPPP1R12A-73aa did not (Fig. 5 c). Moreover, the level of flag-labeled circPPP1R12A-73aa protein was elevated after Lv-OE-flag-circPPP1R12A-73aa or Lv-circPPP1R12A-73aa transfection (Fig. 5 d). We further confirmed that circPPP1R12A-73aa was translated from circPPP1R12A by LC-MS/MS, and the unique aa sequence of circPPP1R12A -73aa was identified. More than 76\% of the circPPP1R12A-73aa sequences were identified in the LC-MS/MS results from the 10-kDa band (Fig. 5 e). In addition, one unique aa sequence formed by the circPPP1R12A "GRLRHVNCLSPGVQD" was identified (Fig. 5 f). Taken together, circPPP1R12A, which was annotated as a circRNA, actually encoded an uncharacterized protein.

\section{CircPPP1R12A-73aa, not circPPP1R12A, promotes the proliferation and metastasis abilities of $\mathrm{CC}$ in vitro and in vivo}

To further explore the biological function of circPPP1R12A-73aa, we generated several cell lines with 

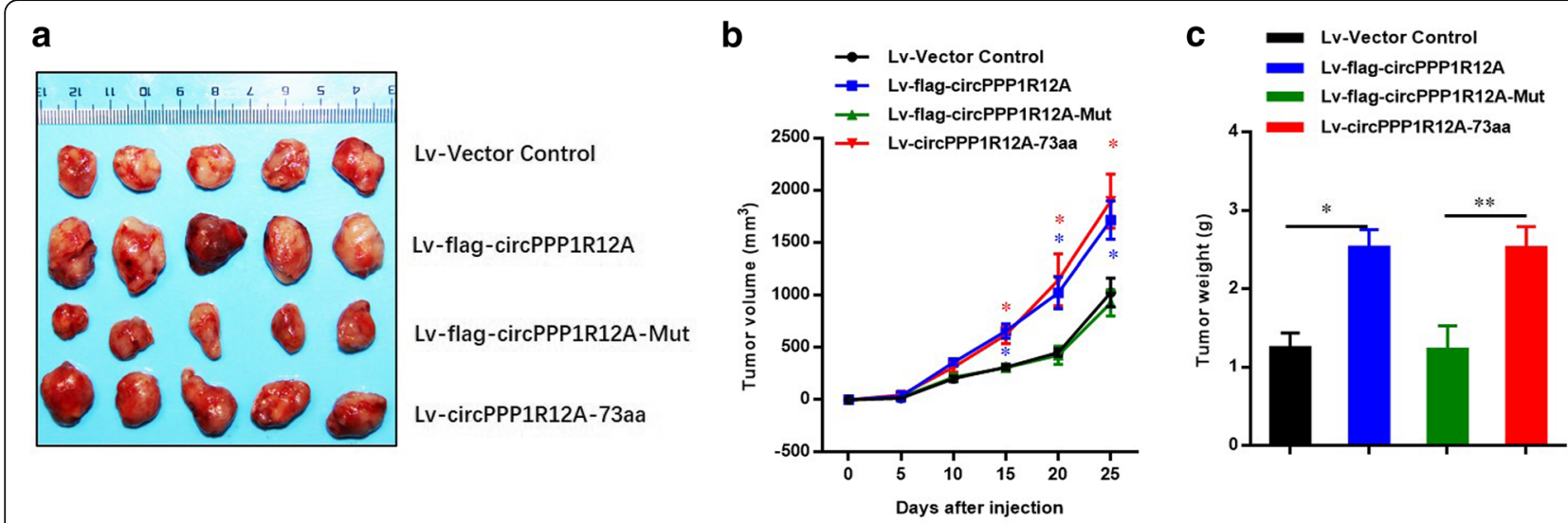

d

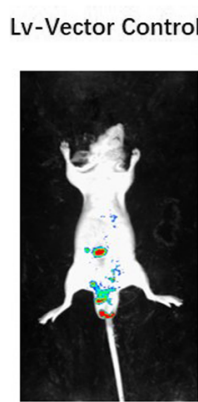

Lv-flag circPPP1R12A

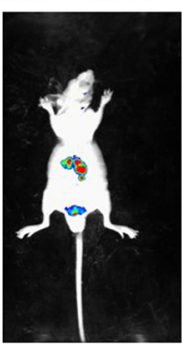

Lv-flag

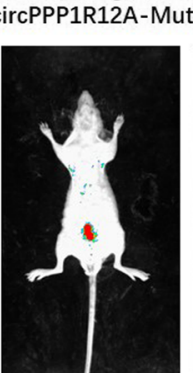

LV

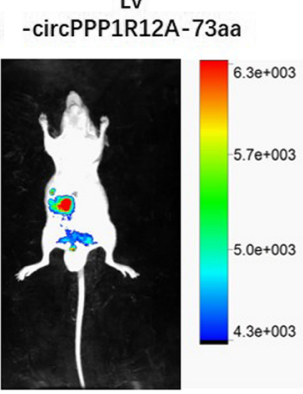

e

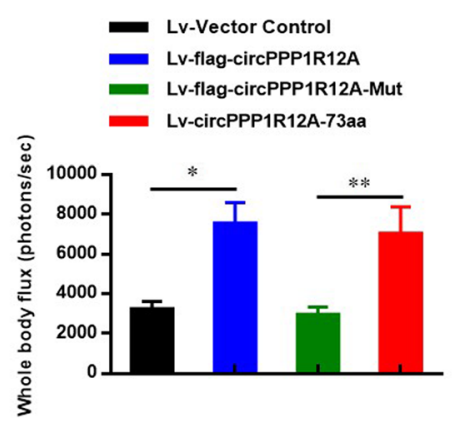

Fig. 7 CircPPP1R12A-73aa, not circPPP1R12A, promotes the in vivo tumorigenicity ability of CC using nude mice xenografts. a Representative nude mice xenograft formed by the indicated cells. b Statistical analysis of xenograft tumor growth formed by the indicated cells. $\mathbf{c}$ Tumor weights of the indicated cells. d Bioluminescent Imaging (BLI) of mice as indicated (Red arrow indicates the metastatic site in BLI of mice). The images were representative of the data. Counts are photons detected. Images were captured with a 5 min exposure. Whole body flux (photons/sec) quantification of mice injected with different $1 \times 10^{5}$ DLD- 1 cells at day $25(n=5)$. The data are represented as the means \pm SEM; ${ }^{*} P<0.05,{ }^{* *} P<0.01,{ }^{* * *} P<0.001$. The red ${ }^{*}$ indicated the LVflag-circPPP1R12A-WT group, The blue * indicated the LV-circPPP1R12A-73aa group

stable transfection of those above-mentioned four vectors (Fig. 5 b). Similar with the previous results (Fig. 3 b), overexpression of wild-type circPPP1R12A with flag labeling resulted in increased proliferation ability, evidenced by CCK8 (Fig. 6 a), colony formation (Fig. 6 b) and cell cycle (Fig. 6 c) assays in DLD-1 and Caco-2 cells. Indeed, only overexpression of circPPP1R12A-73aa increased the proliferation ability too. However, overexpression of mutant circPPP1R12A with flag labeling, which was unable to translate circPPP1R12A-73aa, failed to increase the proliferation ability of DLD-1 and Caco- 2 cells. For the motility of $\mathrm{CC}$ cells, overexpression of circPPP1R12A-73aa increased the migration (Fig. 6 d) and invasion (Fig. 6 e) abilities too, while overexpression of mutant circPPP1R12A failed to increase the migration and invasion abilities of DLD-1 and Caco-2 cells. These data suggested that circPPP1R12A did not regulate the cell proliferation of $\mathrm{CC}$ cells without its encoding protein circPPP1R12A-73aa.

Next, we further investigated the role of circPPP1R12A-73aa in growth of CC cells in vivo using a xenograft tumor model. Figure 7 shows that the tumors generated from cells transfected with Lv-flag-circPPP1R12A and Lv-circPPP1R12A-73aa had a bigger size (Fig. 7 a) and larger weight (Fig. 7 b) compared with those generated from vector control cells. Similar with the in vitro data, the tumors generated from cells transfected with Lv-flag-circPPP1R12A-mut had a smaller size (Fig. 7 a) and lower weight (Fig. 7 c). As the liver metastasis of $\mathrm{CC}$ is the most common metastasis in vivo, we then assessed the effects of circPPP1R12A-73aa on tumor liver metastasis by injecting four stably transfected DLD-1 cells (Fig. 5 b) into the tail veins of nude mice. Subsequently, in vivo bioluminescent imaging (BLI) of mice showed that overexpression of circPPP1R12A-WT and circPPP1R12A-73aa significantly changed the detectable metastasis (red arrow indicates metastasis) in the liver compared with the controls and the circPPP1R12A-Mut group (Fig. $7 \mathrm{~d}$ and e).

Collectively, these data showed that circPPP1R12A-73aa encoded by circPPP1R12A, not circPPP1R12A, promoted the cell proliferation and metastasis of CC cells. 


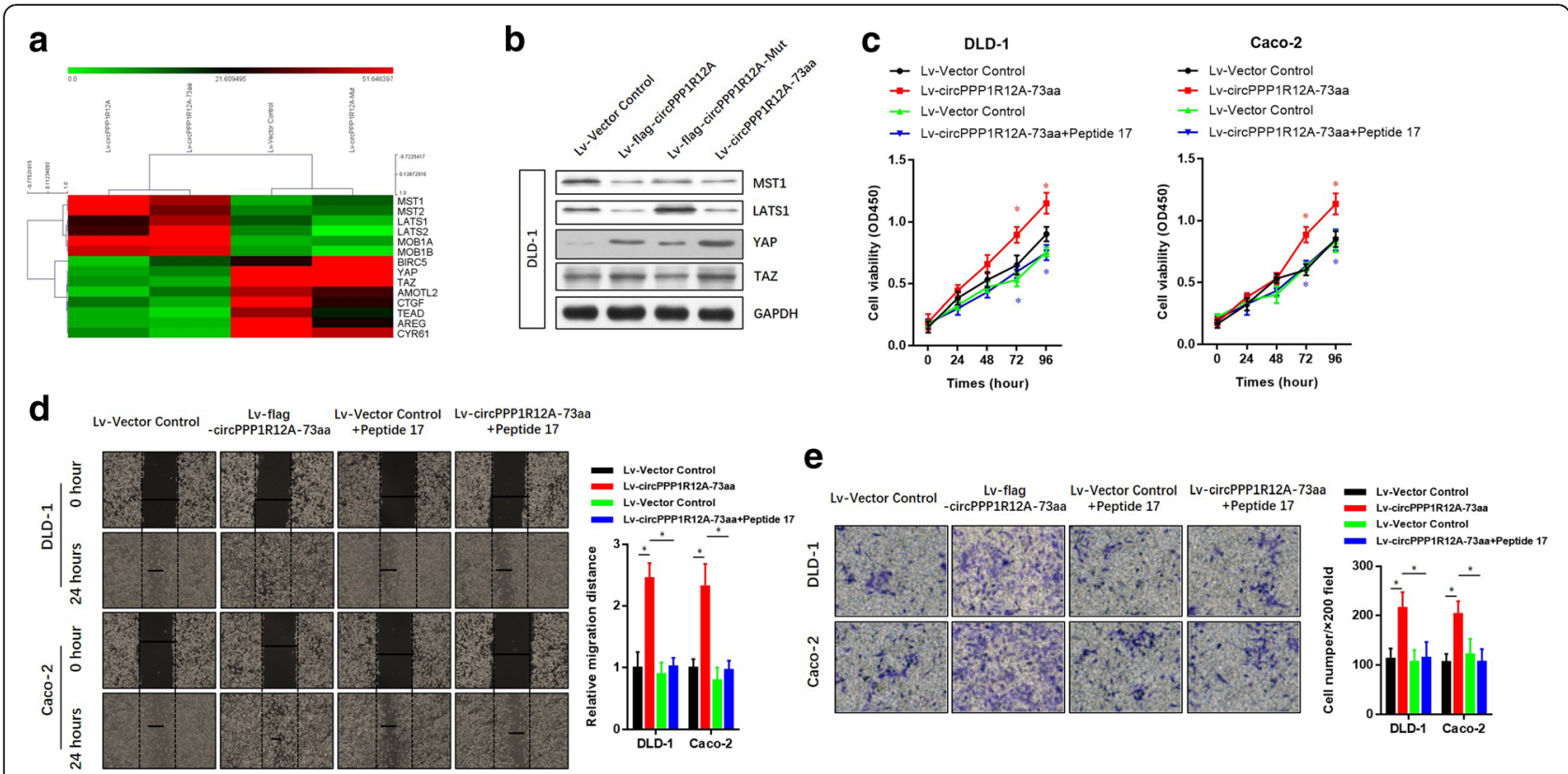

Fig. 8 circPPP1R12A-73aa promotes the proliferation, migration and invasion abilities of CC via activating Hippo-YAP signaling pathway. a heatmap, generated from the RNA-seq analysis, showing differential gene expression between the indicated four groups. b Western Blot was used to evaluate the expression level of MST1, LAST1, YAP and TAZ. c The cell viability was analyzed by CCK8 assay to evaluate the YAP inhibition on the circPPP1R12A73aa promoted proliferation. $\mathbf{d}$ The migration ability was analyzed by wound healing assay to evaluate the YAP inhibition on the circPPP1R12A-73aa promoted proliferation. e The invasion ability was analyzed by transwell assay to evaluate the YAP inhibition on the circPPP1R12A-73aa promoted proliferation. The data are represented as the means $\pm \mathrm{SEM} ;{ }^{*} P<0.05$. The red ${ }^{*}$ indicated the Lv-flag-circPPP1R12A-WT group, The blue * indicated the Lv-circPPP1R12A-73aa group

circPPP1R12A-73aa promotes the proliferation, migration and invasion abilities of CC via activating hippo-YAP signaling pathway

RNA-Seq analyses were carried out to identify the possible signaling pathways, by which circPPP1R12A -73aa promoted the proliferation, migration and invasion. RNA from the four stably transfected DLD cells (Fig. 5 b) was isolated and analyzed. Differentially expressed genes in the four groups were analyzed and enrichened in KEGG pathway database. To elucidate the regulatory mechanism of the signaling influenced by circPPP1R12A-73aa, we focused on the sub-category of signal transduction pathway from the KEGG pathway database. The pathways in this category were ranked, and Fig. 8 a lists the top 10 pathways. As the most enriched pathway, the Hippo-YAP signaling pathway was further proved to be affected by circPPP1R12A-73aa. We next conducted Western blotting analysis to evaluate the dysregulated genes in Hippo-YAP signaling (Fig. 8 b). YAP1 is a transcriptional co-activator in the Hippo signaling pathway, and YAP1-induced transcriptional responses are essential in proliferation and metastasis of cancer cells. To illustrate whether YAP1 activation was critical for the circPPP1R12A-73aa-induced growth and metastasis of cancer cells, we then treated the
circPPP1R12A-73aa-overexpressing DLD-1 and Caco-2 CC cells with YAP1 specific inhibitor Peptide 17. The results revealed that the Peptide 17 significantly alleviated the promotive effect of circPPP1R12A-73aa overexpression on the proliferation (Fig. $8 \mathrm{c}$ ), migration (Fig. $8 \mathrm{~d}$ ) and invasion (Fig. 8 e) abilities of CC cells. Therefore, we confirmed that circPPP1R12A-73aa promoted the proliferation, migration and invasion abilities of CC via activating Hippo-YAP signaling pathway (Fig. 9).

\section{Discussion}

Recently, human genome has been largely transcribed, and non-coding transcripts are found to be abundant in the human transcriptome $[5,23]$. As a type of ncRNAs, circRNAs have recently attracted wide attention $[9,11]$. The broad expression pattern of circRNAs strongly elucidates their roles in regulation of carcinogenesis [13, 24-26]. Recent study has revealed a tumor suppressive protein encoded by the circular form of the SHPRH gene in glioma tumorigenesis [16]. However, it remains unknown whether there are protein-coding circRNAs involved in CC tumorigenesis.

In the present study, we screened the expression profiles of circRNAs between CC tissues and matched adjacent non-tumor tissues, and identified a most elevated circRNA circPPP1R12A in primary cancer 


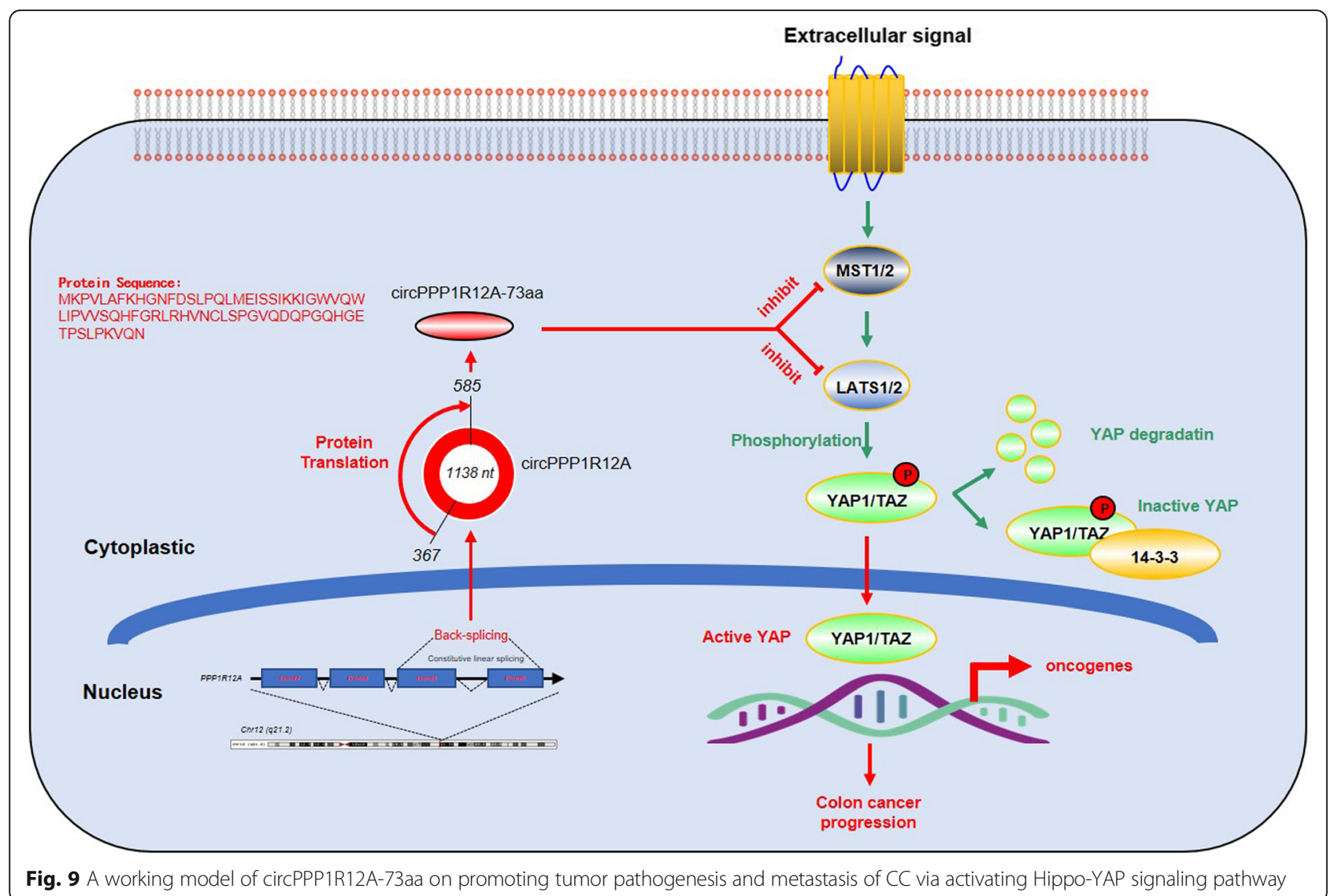

tissues and cancer cell lines. We further provided the evidence that the circRNA circPPP1R12A encoded a conserved 73-aa small peptide, circPPP1R12A-73aa. Moreover, circPPP1R12A-73aa, not circPPP1R12A circRNA itself, promoted the growth and metastasis of CC cells in vitro and in vivo. On the other hand, PPP1R12A, also called the myosin-binding subunit of myosin phosphatase, is one of the subunits of myosin phosphatase, which is frequently expressed at a low level in human cancers. PPP1R12A is mainly involved in the RhoA/ROCK signaling pathway, which participates in the regulation of adhesion, movement, proliferation, differentiation and apoptosis of cells. However, the expression of PPP1R12A was not significantly different between $\mathrm{CC}$ tissues and paired adjacent tissues. In addition, we found that PPP1R12A was expressed at a low level in CC tissues. More studies on alternative splicing during the transcription of PPP1R12A might be carried out to illustrate the regulatory mechanism.

After the earliest characterized circRNA, the sex-determining region of ChrY (Sry), is found [27], cerebellar degeneration-related protein 1 (CDR1as/ciRS-7) has been further demonstrated as an antisense transcript to sponge miR-7 and suppress miR-7 activity [13, 28, 29].
Several cancer-derived circRNAs, including circHIPK3 [18], circMTO1 [12], circCCDC66 [23] and circPVT1 [30], have been reported to be involved in multiple cancers. The functions for circRNAs as well as their underlying mechanisms in carcinogenesis and cancer progression remain largely unexplored. The circRNAs mainly act as ceRNAs, forming circRNA-miRNA-mRNA axis. To the best of our knowledge, circPPP1R12A was the first eukaryotic circRNA encoding small protein in $\mathrm{CC}$, suggesting the complexity of carcinogenesis. The translated product of circPPP1R12A shared most of its aa sequence with that of PPP1R12A, except for the unique aa sequence in the ORF located in the $\mathrm{C}$-terminus. However, the underlying mechanisms by which PPP1R12A-circPPP1R12A-73aa regulated the motility of CC cells needed to be further illustrated.

\section{Conclusions}

Collectively, we found an elevated circRNA circPPP1R12A in the cytoplasm of $\mathrm{CC}$ tissues and cells. In addition, evidence indicated that the circRNA circPPP1R12A encoded a conserved 73-aa small peptide, PPP1R12A-C. The PPP1R12A-C peptide, not circPPP1R12A circRNA itself, promoted the growth of $\mathrm{CC}$ cells in vitro and in vivo. In addition, circPPP1R12A-73aa promoted the proliferation, migration and invasion abilities of $\mathrm{CC}$ cells via activating 
Hippo-YAP signaling pathway. Taken together, our study intuitively illustrated the coding potential of circRNAs in the progression of CC. Moreover, our findings might provide valuable insights into the development of potential therapeutic targets for CC.

\section{Additional files}

Additional file 1: Table S1. Detailed information of the differentially expressed circRNAs. (XLS $66 \mathrm{~kb}$ )

Additional file 2: Figure S1. PPP1R12A expression in CC tissues and cells. a The expression level of PPP1R12A in CC and matched non-tumor tissue samples from 20 patients was analyzed by real-time PCR. $\mathbf{b}$ The expression level of PPP1R12A in a series of cultured CC cell lines (HT-29, HCT-116, SW480, SW620, LoVo, SW48, DLD-1, Caco2 and HCT-15) was analyzed by real-time PCR. (JPG $50 \mathrm{~kb}$ )

Additional file 3: Figure S2. Circular RNA circPPP1R12A promotes the cell proliferation, but not migration and invasion abilities of HCT-116 and LoVo colon cancer cells. a circPPP1R12A promotes the proliferation of HCT-116 and LoVo cells shown by CCK8 assay. $\mathbf{b}$ circPPP1R12A promotes the proliferation of HCT-116 and LoVo cells shown by colony formation assay. c circPPP1R12A did not affect the migration of HCT-116 and LoVo cells shown by wound healing assay. $\mathbf{d}$ circPPP1R12A did not affect the invasion of HCT-116 and LoVo cells shown by matrial assay. The data are represented as the means $\pm \mathrm{SEM}$; ${ }^{*} P<0.05$, N.S, not significant. (JPG $402 \mathrm{~kb})$

\section{Abbreviations}

circRNAs: Circular RNAs; FISH: fluorescence in situ hybridization; ISH: in situ hybridization; ORF: open reading frame

\section{Acknowledgements}

We thank all patients involved in this study.

\section{Funding}

This work was supported by grants from the National Key R\&D Plan (2018YFC1313400), the National Natural Science Foundation of China (31570877, 31570908, 31729001, 31701111), the National Key Technology R\&D Program (2015BAl12B12), Jiangsu Engineering Research Center for Tumor Immunotherapy (BM2014404), the Key R\&D Project of Science and Technology Department of Jiangsu Province (BE2018645, BE2015633, BE2016660), Young Medical Talents Program of Jiangsu Province (QNRC2016286), Jiangsu Key Laboratory of Medical Science and Laboratory Medicine (JSKLM-2014-003), Changzhou Science and Technology Project (Applied Based Research, CJ20160021, CJ20179048) and Changzhou HighLevel Medical Talents Training Project (2016CZBJ001).

\section{Availability of data and materials}

All data in our study are available upon request.

\section{Authors' contributions}

$X$. Zheng and JT. Jiang designed this study. X. Zheng performed all experiments; $L$. Chen and Y. Zhou collected tissue samples and the clinical data; Q. Wang and B. Xu analyzed and interpreted the data; X. Zheng, ZJ. Zheng, C. Wu, Q. Zhou, WW. Hu and CP. Wu drafted the manuscript. All authors read and approved the final manuscript.

\section{Ethics approval and consent to participate}

Written informed consent for the biological studies was obtained from each patient involved in the study, and the study was approved by the Ethics Committee of the Third Affiliated Hospital of Soochow University. All animal studies were approved by the Animal Experimental Committee of the Third Affiliated Hospital of Soochow University.

\section{Consent for publication}

Written consents for publication were obtained from all the patients involved in our study.

\section{Competing interests}

The authors declare no conflict of interest.

\section{Publisher's note}

Springer Nature remains neutral with regard to jurisdictional claims in published maps and institutional affiliations.

\section{Author details}

'Department of Tumor Biological Treatment, the Third Affiliated Hospital of Soochow University, Changzhou 213003, People's Republic of China. ${ }^{2}$ Jiangsu Engineering Research Center for Tumor Immunotherapy, Changzhou 213003, People's Republic of China. Institute of Cell Therapy, Soochow University, Changzhou 213003, People's Republic of China. ${ }^{4}$ Department of Hematology, the Third Affiliated Hospital of Soochow University, Changzhou 213003, People's Republic of China. ${ }^{5}$ Department of Oncology, the Third Affiliated Hospital of Soochow University, Changzhou 213003, People's Republic of China.

Received: 31 August 2018 Accepted: 25 March 2019

Published online: 29 March 2019

\section{References}

1. Cassidy S, Syed BA. Colorectal cancer drugs market. Nat Rev Drug Discov. 2017;16:525-6.

2. Cressman S, Browman GP, Hoch JS, Kovacic L, Peacock SJ. A time-trend economic analysis of Cancer drug trials. Oncologist. 2015;20:729-36.

3. Pan Y, Tong JHM, Lung RWM, Kang W, Kwan JSH, Chak WP, Tin KY, Chung LY, Wu F, Ng SSM, Mak TWC, Yu J, Lo KW, Chan AWH, To KF: RASAL2 promotes tumor progression through LATS2/YAP1 axis of hippo signaling pathway in colorectal cancer. Mol Cancer 2018;17:102.

4. Valentovic MA. Evaluation of resveratrol in Cancer patients and experimental models. Adv Cancer Res. 2018;137:171-88.

5. Adelman K, Egan E. Non-coding RNA: more uses for genomic junk. Nature. 2017;543:183-5.

6. Duss O, Michel E, Yulikov M, Schubert M, Jeschke G, Allain FH. Structural basis of the non-coding RNA RsmZ acting as a protein sponge. Nature. 2014;509:588-92.

7. Hermann T. Non-coding RNA: antibiotic tricks a switch. Nature. 2015;526: 650-1.

8. Kleaveland B, Shi CY, Stefano J, Bartel DP: A network of noncoding regulatory RNAs acts in the mammalian brain. Cell 2018;174:350-362 e317.

9. Memczak S, Jens M, Elefsinioti A, Torti F, Krueger J, Rybak A, Maier L, Mackowiak SD, Gregersen LH, Munschauer M, Loewer A, Ziebold U, Landthaler M, Kocks C, le Noble F, Rajewsky N. Circular RNAs are a large class of animal RNAs with regulatory potency. Nature. 2013;495:333-8.

10. Ledford H. Circular RNAs throw genetics for a loop. Nature. 2013;494:415.

11. Vicens Q, Westhof E. Biogenesis of circular RNAs. Cell. 2014;159:13-4.

12. Han D, Li J, Wang H, Su X, Hou J, Gu Y, Qian C, Lin Y, Liu X, Huang M, Li N, Zhou W, Yu Y, Cao X. Circular RNA circMTO1 acts as the sponge of microRNA-9 to suppress hepatocellular carcinoma progression. Hepatology. 2017;66:1151-64.

13. Hansen $T B$, Jensen $T I$, Clausen BH, Bramsen JB, Finsen B, Damgaard CK, Kjems J. Natural RNA circles function as efficient microRNA sponges. Nature. 2013:495:384-8.

14. Du WW, Yang W, Li X, Awan FM, Yang Z, Fang L, Lyu J, Li F, Peng C, Krylov SN, Xie Y, Zhang Y, He C, Wu N, Zhang C, Sdiri M, Dong J, Ma J, Gao C, Hibberd S, Yang BB. A circular RNA circ-DNMT1 enhances breast cancer progression by activating autophagy. Oncogene. 2018. https://doi.org/10. 1038/s41388-018-0369-y.

15. Kristensen LS, Hansen TB, Veno MT, Kjems J. Circular RNAs in cancer: opportunities and challenges in the field. Oncogene. 2018;37:555-65.

16. Zhang M, Huang N, Yang X, Luo J, Yan S, Xiao F, Chen W, Gao X, Zhao K, Zhou H, Li Z, Ming L, Xie B, Zhang N. A novel protein encoded by the circular form of the SHPRH gene suppresses glioma tumorigenesis. Oncogene. 2018;37:1805-14.

17. Matsumoto A, Nakayama Kl. Hidden peptides encoded by putative noncoding RNAs. Cell Struct Funct. 2018;43:75-83.

18. Zheng Q, Bao C, Guo W, Li S, Chen J, Chen B, Luo Y, Lyu D, Li Y, Shi G, Liang L, Gu J, He X, Huang S. Circular RNA profiling reveals an abundant circHIPK3 that regulates cell growth by sponging multiple miRNAs. Nat Commun. 2016;7:11215. 
19. Qiu M, Xia W, Chen R, Wang S, Xu Y, Ma Z, Xu W, Zhang E, Wang J, Fang T, Hu J, Dong G, Yin R, Wang J, Xu L. The circular RNA circPRKCl promotes tumor growth in Lung adenocarcinoma. Cancer Res. 2018;78:2839-51.

20. Wagner F, Streubel A, Roth A, Stephan-Falkenau S, Mairinger T.

Chromogenic in situ hybridisation (CISH) is a powerful method to detect ALK-positive non-small cell lung carcinomas. J Clin Pathol. 2014;67:403-7.

21. Chen L, Zhai W, Zheng X, Xie Q, Zhou Q, Tao M, Zhu Y, Wu C, Jiang J. Decreased IFIT2 expression promotes gastric Cancer progression and predicts poor prognosis of the patients. Cell Physiol Biochem. 2018;45:15-25.

22. Ferreira R, Trindade F, Vitorino R. Proteome profiling of Sertoli cells using a GeLC-MS/MS strategy. Methods Mol Biol. 1748;2018:173-90.

23. Hsiao KY, Lin YC, Gupta SK, Chang N, Yen L, Sun HS, Tsai SJ. Noncoding effects of circular RNA CCDC66 promote Colon Cancer growth and metastasis. Cancer Res. 2017;77:2339-50.

24. Zhou R, Wu Y, Wang W, Su W, Liu Y, Wang Y, Fan C, Li X, Li G, Li Y, Xiong W, Zeng Z. Circular RNAs (circRNAs) in cancer. Cancer Lett. 2018;425:134-42.

25. Yang C, Yuan W, Yang X, Li P, Wang J, Han J, Tao J, Li P, Yang H, Lv Q, Zhang W. Circular RNA circ-ITCH inhibits bladder cancer progression by sponging miR-17/miR-224 and regulating p21 PTEN expression. Mol Cancer. 2018;17:19.

26. Zhang J, Liu H, Hou L, Wang G, Zhang R, Huang Y, Chen X, Zhu J. Circular RNA_LARP4 inhibits cell proliferation and invasion of gastric cancer by sponging miR-424-5p and regulating LATS1 expression. Mol Cancer. 2017; 16:151.

27. Capel B, Swain A, Nicolis S, Hacker A, Walter M, Koopman P, Goodfellow P, Lovell-Badge R. Circular transcripts of the testis-determining gene Sry in adult mouse testis. Cell. 1993;73:1019-30.

28. Sang M, Meng L, Sang Y, Liu S, Ding P, Ju Y, Liu F, Gu L, Lian Y, Li J, Wu Y, Zhang $X$, Shan B. Circular RNA ciRS-7 accelerates ESCC progression through acting as a miR-876-5p sponge to enhance MAGE-A family expression. Cancer Lett. 2018;426:37-46.

29. Xu L, Zhang M, Zheng X, Yi P, Lan C, Xu M. The circular RNA ciRS-7 (Cdr1as) acts as a risk factor of hepatic microvascular invasion in hepatocellular carcinoma. J Cancer Res Clin Oncol. 2017;143:17-27.

30. Chen J, Li Y, Zheng Q, Bao C, He J, Chen B, Lyu D, Zheng B, Xu Y, Long Z, Zhou Y, Zhu H, Wang Y, He X, Shi Y, Huang S. Circular RNA profile identifies circPVT1 as a proliferative factor and prognostic marker in gastric cancer. Cancer Lett. 2017;388:208-19.

Ready to submit your research? Choose BMC and benefit from:

- fast, convenient online submission

- thorough peer review by experienced researchers in your field

- rapid publication on acceptance

- support for research data, including large and complex data types

- gold Open Access which fosters wider collaboration and increased citations

- maximum visibility for your research: over $100 \mathrm{M}$ website views per year

At $\mathrm{BMC}$, research is always in progress.

Learn more biomedcentral.com/submissions 